\title{
Narcolepsy: A Chronic Neurological Disorder - A Review
}

\author{
Aanchal Ajit Jain \\ First year student of Datta Meghe Medical College
}

\section{ABSTRACT}

The deficiency or dysfunction of orexin (hypocretin) neurons in the lateral hypothalamus causes narcolepsy, a rare neurological condition. Narcolepsy is one of the most molecularly researched sleep disorders. Narcolepsy impacts between 0.02-0.05 percent of the world's population, according to estimates. Excessive daytime sleepiness and cataplexy are signs of narcolepsy type 1 (NT1), which are followed by sleep-wake symptoms such as hallucinations, sleep paralysis, and disrupted sleep. Type 1 narcolepsy is due to poor Brain and Nervous system (CNS) hypocretin signaling, which is distinguishable from other primary CNS hypersomnia, which appears to lie along a range of narcolepsy Type 2 to undiagnosed hypersomnia. It's marked by extreme daytime sleepiness and a lack of muscle tone (cataplexy). The information collected affirm the view of NT1 as a hypothalamic condition involving not only sleep-wake but also sensory, psychological, mental, cognitive, biochemical, and somatic processes, as well as uncertainties about the 'narcoleptic borderland,' including narcolepsy form 2 (NT2). Medications are currently symptom-based, although they have seen mixed outcomes as applied to other hypersomnia's. The diagnosis of narcolepsy is based on clinical symptoms and the exclusion of other factors of excessive sleepiness. Stimulant medications are used to treat daytime sleepiness, numb hypnotics for cataplexy, and hydroxybutyrate for both symptoms. Since narcolepsy is such an under-diagnosed condition, it's critical for healthcare professionals and other primary health-care providers to spot unexplained daytime sleepiness early on.

KEY WORDS: NARCOLEPSY, CATAPLEXY, SLEEP DISORDER, OREXIN/HYPOCRETIN, EXCESSIVE DAYTIME SLEEPINESS, SLEEP PARALYSIS, MULTIPLE SLEEP LATENCY TEST, POLYSOMNOGRAPHY, RAPID EYE MOVEMENT.

\section{INTRODUCTION}

Any person with narcolepsy experiences extreme daytime sleepiness (EDS) and a proclivity to doze off. Atypical Rapid Eye Movement (REM) sleep is also an effect. Dreaming and muscular dysfunction are common symptoms of REM, which prevents people from acting out their fantasies (Overeem et al., 2001). REM sleep can occur at any stage of day in narcolepsy, and components of rem sleep can combine with wakefulness, resulting in hypnopompic and hypnagogic hallucination, as well as sleep paralysis. Cataplexy is a discrete symptom that is caused by intense emotions or thoughts.

Biosc Biotech Res Comm P-ISSN: 0974-6455 E-ISSN: 2321-4007

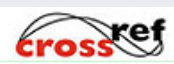

Identifiers and Pagination

Year: 2021 Vol: 14 No (6) Special Issue

Pages: 289-292

This is an open access article under Creative

Commons License Attribn 4.0 Intl (CC-BY).

DOI: $h t t p: / / d x . d o i . o r g / 10.21786 / b b r c / 14.6 .61$

\section{Narcolepsy is of Two Types:}

Narcolepsy type 1 (was also known as narcolepsy with cataplexy). Type 1 narcolepsy is characterized by excessive daytime sleepiness with cataplexy, and/or reduced levels of hypocretin, a neurotransmitter.

Narcolepsy type 2 (was also known as narcolepsy without cataplexy). Type 2 narcolepsy is characterized by excessive daytime sleepiness without cataplexy and normal hypocretin levels.

\section{Signs and Symptoms}

Cataplexy: Cataplexy is a symptom of narcolepsy type 1 that causes transient muscle fatigue caused by emotion. It usually starts in the facial muscles and manifests as a slackening of the jaw or a brief head falling. However, episodes can be more severe, resulting in crashing down unless the trunk and limb structures are compromised. Cataplexy normally appears around the same period as narcolepsy-related sleepiness, but it may also appear

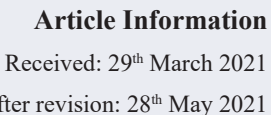

ccepted after revision: $28^{\text {th }}$ May 2021 c) creative cointions 
years later (Scammell, 2003; Golden et al., 2008; Dauvilliers et al., 2007).

The length of each episode varies from a few seconds to two minutes. Consciousness is maintained at all times. Cataplexy may be triggered by a variety of feelings, but it is most often a happy emotion like laughter or enthusiasm. Cataplexy is common in people with narcolepsy; however, not all with narcolepsy can have cataplexy.

Excessive daytime sleepiness (EDS): Daytime sleepiness is usually the first symptom to occur. It's both the most serious symptom and the most common reason for a doctor's visit (Dauvilliers et al., 2001). Daytime sleepiness occurs on a regular basis, with recurrences usually every 2 hours, though this can vary. Narcolepsy is characterized clinically by EDS which can disable a person personally and socially. Inactivity makes sleepiness harder, and sleep can be difficult to avoid. Sleep attacks can happen unexpectedly, and they can last long enough to trigger a lapse in consciousness. Short naps are known to be rejuvenating. In narcolepsy, the REM duration- the time from falling unconscious to the beginning of REM sleep-is low, and though the REM stage is when dreams arise, naps often involve dreaming. As a result, it's worth asking patients whether they sleep through naps before taking a history; a yes response confirms the diagnosis of narcolepsy. Daytime sleepiness is common in people with narcolepsy (Scammell, 2015).

Hypnagogic hallucinations: Hypnagogic or hypnopompic (on awakening) hallucinations may be sensory, visual, or somesthetic. Such dreams can be so disturbing that the person becomes afraid of going to bed and returns to reassuring behaviors such as letting their pet sleep in the same room with them (Plazzi et al., 2015; Hishikawa and Shimizu, 1995). And if patients are mindful of the existence of these phenomena in retrospect, some patients report difficulties distinguishing hallucinations from reality and can occasionally be masked.

Sleep Paralysis: You can feel paralyzed and unable to move or speak just when you are falling asleep or waking up. This is referred to as sleep paralysis. The failure to react or speak is just temporary, lasting for more than a couple of minutes. This can occur simultaneously with a hallucination. Once the sleep paralysis has passed, you should have no trouble moving or speaking. The most common moment for sleep paralysis to occur is when you first wake up, but it can also happen right before you fall asleep. It usually manifests itself as an inability to lift the arms or talk for a few seconds or, in extreme situations, minutes at a time. Sleep paralysis may be associated with feelings of anxiety or suffocation, particularly when it occurs for the first time.

Disrupted Sleep: Studies have shown that narcoleptic patients hardly sleep at night time, which is usually interrupted in one-third of patients when they are unable to remain awake or are unconscious for extended duration of time. Patients usually fall asleep as soon as they get into bed, but they get up several times in the night. Narcoleptic patients have higher body mass index than those who do not have this disorder (Ankar et al., 2020). Following the onset of narcolepsy, children specifically gain weight.

\section{Diagnosis}

Actigraphy and polysomnography testing Actigraphy: Actigraphy is a noninvasive, straightforward tool for tracking cumulative sleep time (TST) over weeks or months. It is based on the theory that when people are alive, they shift their wrists almost constantly, while when they sleep, they move their wrists only sometimes (Nesbitt, 2018). Polysomnography is the next move if actigraphy shows the patient is keeping a daily sleep routine and having enough time for nighttime sleep.

Polysomnography: A sleep analysis, also known as polysomnography (PSG), is a structured process used to identify sleep disorders. This method is used to see whether you have narcolepsy or idiopathic hypersomnia. The purpose of the PSG is to rule out other causes of daytime sleepiness (most notably sleep apnea) and determine whether the patient has gotten enough sleep (at least 6 hours) prior to the MSLT. Furthermore, polysomnography may reveal a relatively short REM sleep latency (less than 15 minutes) in 40 percent of cases, REM fragmentation with muscle tone loss, stage 1 sleep increased proportions, and a comparative enhancement in gradual sleep at the end of the night (Dauvilliers et al., 2007; Scammell, 2015).

Multiple Sleep Latency Test: This test is normally performed the next day after your overnight sleep analysis is completed, basically it is the next step of PSG. The MSLT consists of five 2-hourly nap times that begin at least 15 hours after awakening. If the patient does not fall asleep after 15 minutes, the test is terminated. The occurrence of REM sleep within 15 minutes of sleep onset is referred to as a sleep-onset REM period (SOREMP). However, since the multiple sleep latency test has a low sensitivity, it should be repeated if narcolepsy is suspected. It's also not entirely accurate, and falsepositive results will occur (Darien, 2014). SOREMPs can be found in the general population, especially in people who suffer from a circadian rhythm disorder, inadequate sleep, or sleep-disordered breathing. Just a small percentage of the general population shows two or more SOREMPs in MSLT. MSLT findings should be viewed in a clinical setting.

Differential Diagnostics: Idiopathic hypersomnia, another type of central hypersomnolence, is characterized by incapacitating sleepiness. Since there are less than two SOREMPs, it can be distinguished from narcolepsy. In contrast to narcolepsy, where naps are often refreshing, naps in idiopathic hypersomnia are often ineffective at restoring wakefulness. Sleep is normally not fragmented in idiopathic hypersomnia, and there are few nocturnal arousals. Patients with idiopathic hypersomnia are sleepy during the day and have a shorter MSLT, but they do not enter REM pathologically rapidly. 
Kleine-Levin syndrome is a hypersomnia condition that is rare. When in contrast with the chronic sleepiness of narcolepsy and idiopathic hypersomnia, it is anecdotal. Hypersomnia lasts for days to weeks at a time and is followed by cognitive and behavioural changes such as hyperphagia and hypersexuality (Jain et al., 2018; Arnulf et al., 2005).

Sleep apnoea syndrome is often confused with MSLT, which is a significant cause of daytime sleepiness (usually in middle-aged rather than young people) but never triggers cataplexy. Night-time polysomnography is a simple method to diagnose it.

About orexin levels: A hypocretin deficiency in the central nervous system has been attributed to type 1 narcolepsy. Hypocretin (also known as orexin) is a hormone released in the hypothalamus that regulates alertness by acting on multiple brain regions (Nishino et al., 2000). Hypothalamic neurons that produce hypocretin are selectively decreased in narcolepsy type 1 for unknown reasons. Hypocretin also regulates wakefulness and prevents REM sleep, so a lack of it can trigger REM sleep intrusions into wakefulness, resulting in cataplexy, sleep-related hallucinations, and sleep paralysis, which are all hallmarks of narcolepsy (Chaturvedi et al., 2020). Hypocretin deficiency is caused by the lack of hypocretin neurons in the dorsolateral hypothalamus in people with narcolepsy with cataplexy.

Autoimmunity Basis: While family cases have been reported, narcolepsy is usually a sporadic condition. A parent with narcolepsy has a one-in-ten chance of having a child with the disorder.

HLA-DQB1*0602 allele is closely linked to narcolepsy type 1, with up to 95 percent of those affected carrying at least one allele (Mignot et al.,1997). The risk of developing narcolepsy is increased even further if you have two copies of the allele. This mutation, however, is not unique to narcolepsy with cataplexy, as it is found in $12 \%$ to $38 \%$ of the general population. As a result, HLA typing has minimal clinical usefulness at the moment (Akintomide et al., 2011; Pelin et al., 1998). There was a large rise in the incidence of narcolepsy with cataplexy following the $2009 \mathrm{H} 1 \mathrm{~N} 1$ influenza pandemic, reigniting interest in an autoimmune etiology. An rise in the occurrence of narcolepsy was also linked to Pandemrix, an H1N1 vaccine developed in response to the 2009 pandemic (Mahoney et al., 2019). There has also been a connection to other upper respiratory infections, implying an autoimmune origin.

Treatment: Narcolepsy does not have a cure; if you have it, you will always have it. However, there are several treatment options available. Lifestyle modifications are the first step in managing narcolepsy symptoms. Modafinil is a sedative that promotes alertness. Despite the fact that the drug's mechanism of action is unknown, it is prescribed as a first-line treatment for narcolepsy for many reasons. Methylphenidate is a powerful stimulant that is widely used. It specifically inhibits the reuptake of monoamines (primarily dopamine) and does not inhibit the vesicular monoamine transporter, unlike amphetamines (Golden et al., 2011; Dauvilliers et al., 2007). Another drug that can help you with cataplexy and excessive daytime sleepiness is sodium oxybate.

\section{CONCLUSION}

Narcolepsy has no known cure. It's a sleep disorder that lasts a lifetime. It does not, generally get worse with age. Medications and lifestyle changes can help to alleviate the symptoms of narcolepsy. The standardised narcolepsy criteria and diagnostic tests are widely recognised, but they will need to be revisited in the future.

\section{REFERENCES}

Akintomide, G.S. and Rickards, H., 2011. Narcolepsy: a review. Neuropsychiatric disease and treatment, 7, p.507.

American Academy of Sleep Medicine. International Classification of Sleep Disorders. 3rd ed. Darien, IL: American Academy of Sleep Disorders; 2014.

Ankar, Shrikant, R., Singh, S., 2020. Chemotherapy Induced Peripheral Neuropathy - A Review. JOURNAL OF EVOLUTION OF MEDICAL AND DENTAL SCIENCESJEMDS, 9(42), pp. 3147-51.

Arnulf, I., Zeitzer, J.M., File, J., Farber, N. and Mignot, E., 2005. Kleine-Levin syndrome: a systematic review of 186 cases in the literature. Brain, 128(12), pp.27632776.

Carskadon, M.A., 1986. Guidelines for the multiple sleep latency test (MSLT): a standard measure of sleepiness. Sleep, 9(4), pp.519-524.

Chaturvedi, A., Garikapati, A., Bagga, C.S., Chande, A. and Kumar, S., 2020. Temporal lobe epilepsy: A rare post chicken pox neurological sequel. Medical Science, 24(103), pp.1596-1599.

Dauvilliers, Y., Arnulf, I. and Mignot, E., 2007. Narcolepsy with cataplexy. The Lancet, 369(9560), pp.499-511.

Dauvilliers, Y., Montplaisir, J., Cochen, V., Desautels, A., Einen, M., Lin, L., Kawashima, M., Bayard, S., Monaca, C., Tiberge, M. and Filipini, D., 2010. Post-H1N1 narcolepsy-cataplexy.

Dauvilliers, Y., Montplaisir, J., Molinari, N., Carlander, B., Ondze, B., Besset, A. and Billiard, M., 2001. Age at onset of narcolepsy in two large populations of patients in France and Quebec. Neurology, 57(11), pp.20292033.

Golden, E.C., Lipford, M.C., 2018. Narcolepsy: Diagnosis and management. Cleve Clin J Med.,85(12), pp.959969.

Hishikawa, Y. and Shimizu, T., 1995. Physiology of REM sleep, cataplexy, and sleep paralysis. Advances in neurology, 67, pp.245-271.

Jain, J., Banait, S., Tiewsoh, I. and Choudhari, M., 2018. Kikuchi's disease (histiocytic necrotizing lymphadenitis): A rare presentation with acute kidney injury, peripheral 
neuropathy, and aseptic meningitis with cutaneous involvement. Indian Journal of Pathology and Microbiology, 61(1), p.113.

Latchoumi, T.P., Ezhilarasi, T.P. and Balamurugan, K., 2019. Bio-inspired weighed quantum particle swarm optimization and smooth support vector machine ensembles for identification of abnormalities in medical data. SN Applied Sciences, 1(10), pp.1-10.

Mahoney, C.E., Cogswell, A., Koralnik, I.J. and Scammell, T.E., 2019. The neurobiological basis of narcolepsy. Nature Reviews Neuroscience, 20(2), pp.83-93.

Mignot, E., Hayduk, R., Black, J., Grumet, F.C. and Guilleminault, C., 1997. HLA DQB1* 0602 is associated with cataplexy in 509 narcoleptic patients. Sleep, 20(11), pp.1012-1020.

Nesbitt, A.D., 2018. Delayed sleep-wake phase disorder. Journal of thoracic disease, 10(Suppl 1), p.S103.

Nishino, S., Ripley, B., Overeem, S., Lammers, G.J. and Mignot, E., 2000. Hypocretin (orexin) deficiency in human narcolepsy. The Lancet, 355(9197), pp.39-40.

Overeem, S., Mignot, E., GertvanDijk, J. and Lammers,
G.J., 2001. Narcolepsy: clinical features, new pathophysiologic insights, and future perspectives. Journal of Clinical Neurophysiology, 18(2), pp.78105.

Pelin, Z., Guilleminault, C., Risch, N., FC Grumet US Modafinil in Narcolepsy Multicenter Study Group and Mignot, E., 1998. HLA-DQB1* 0602 homozygosity increases relative risk for narcolepsy but not disease severity in two ethnic groups. Tissue antigens, 51(1), pp.96-100.

Plazzi, G., Fabbri, C., Pizza, F. and Serretti, A., 2015. Schizophrenia-like symptoms in narcolepsy type 1: shared and distinctive clinical characteristics. Neuropsychobiology, 71(4), pp.218-224.

Scammell, T.E., 2003. The neurobiology, diagnosis, and treatment of narcolepsy. Annals of Neurology: Official Journal of the American Neurological Association and the Child Neurology Society, 53(2), pp.154-166.

Scammell, T.E., 2015. Narcolepsy. New England Journal of Medicine, 373(27), pp.2654-2662. 